RECOMMENDATIONS

\title{
Recommendations for the anticoagulation of pregnant patients with mechanical heart valves
}

\begin{abstract}
E Schapkaitz, B F Jacobson, P Manga, R S Chitsike, E Benade, S Jackson, S Haas, H R Buller, on behalf of the South African Society of Thrombosis and Haemostasis

Dr Elise Schapkaitz is a haematologist at Charlotte Maxeke Johannesburg Academic Hospital and the Department of Molecular Medicine and Haematology, Faculty of Health Sciences, University of the Witwatersrand, Johannesburg, South Africa; Prof. Barry Jacobson is Head of the Division of Clinical Haematology at Charlotte Maxeke Johannesburg Academic Hospital and the Faculty of Health Sciences, University of the Witwatersrand, and chairman of the South African Society of Thrombosis and Haemostasis; Prof. Pravin Manga is Head of the Division of Cardiology at Charlotte Maxeke Johannesburg Academic Hospital and the Faculty of Health Sciences, University of the Witwatersrand; Dr Rufaro Chitsike is a clinical haematologist at Medicine Memorial University of Newfoundland Health Sciences Center, Newfoundland, Canada; Dr Estee Benade is a senior haematology registrar at Charlotte Maxeke Johannesburg Academic Hospital and the Faculty of Health Sciences, University of the Witwatersrand; Dr Sarah Jackson is an obstetrician and gynaecologist at Charlotte Maxeke Johannesburg Academic Hospital and the Faculty of Health Sciences, University of the Witwatersand; Prof. Sylvia Haas is a clinical haematologist at the Technical University of Munich, Germany, and an international expert in the field of thrombosis and haemostasis; and Prof. H R Buller, an international expert in the same field, is a clinical haematologist at the Academic Medical Centre, University of Amsterdam, The Netherlands.
\end{abstract}

Corresponding author: E Schapkaitz (elise.schapkaitz@nhls.ac.za)

Background. The management of pregnant patients with mechanical heart valves remains challenging. Both vitamin $\mathrm{K}$ antagonists and heparins may be associated with maternal and fetal adverse events.

Method. The Southern African Society of Thrombosis and Haemostasis reviewed available literature and comprehensive evidence-based guidelines for the anticoagulation of pregnant patients with mechanical heart valves. A draft document was produced and revised by consensus agreement. The recommendations were adjudicated by independent international experts to avoid local bias.

Results and conclusion. We present concise, practical guidelines for the clinical management of pregnant patients with mechanical heart valves. Recommendations reflect current best practice, which it is hoped will lead to improved anticoagulation practice in this select group of high-risk patients.

S Afr Med J 2015;105(9):733-738. DOI:10.7196/SAMJnew.7928

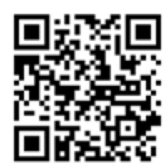

\section{Introduction}

Pregnancy in patients with mechanical heart valves is associated with considerable morbidity, mortality and health cost utilisation. Management should be individualised in consultation with a multidisciplinary team including an obstetrician, a cardiologist and a haematologist to undertake laboratory monitoring, with appropriate consideration for the risk/benefit ratio of each patient. We have designed practice guidelines to assist doctors and nurses caring for pregnant women with mechanical heart valves, with a view to achieving improved anticoagulation practice in this select group of high-risk patients.

\section{Methodology}

On behalf of the Southern African Society of Thrombosis and Haemostasis, a representative guideline panel of professionals from the fields of haematology, cardiology and obstetrics reviewed the available English language literature on the anticoagulation of pregnant patients with mechanical heart valves on Pubmed. This included systematic reviews and randomised controlled trials as well as case series, observational studies and cohort studies. In addition, comprehensive evidence-based guidelines, namely the 9th edition of the American College of Chest Physicians (ACCP) and American Heart Association (AHA) guidelines, ${ }^{[1,2]}$ were consulted and referenced where applicable. A draft document was produced and revised by consensus agreement. These resulting recommendations were adjudicated and co-authored by independent international experts to avoid local bias.

The recommendations have been graded as strong (grade 1) or weak (grade 2) according to the risks, adverse effects, health benefits and cost (Table 1).
Table 1. Grading recommendation of the ACCP for antithrombotic therapy ${ }^{[1]}$

\begin{tabular}{ll}
\hline Grade & Methodological quality of supporting evidence \\
\hline 1A & $\begin{array}{l}\text { Consistent evidence from RCTs without important limitations, or exceptionally } \\
\text { strong evidence from observational studies }\end{array}$ \\
1B $\quad \begin{array}{l}\text { Evidence from RCTs with important limitations (inconsistent results, methodological } \\
\text { flaws), or very strong evidence from observational studies }\end{array}$ \\
1C $\quad \begin{array}{l}\text { Evidence for at least one critical outcome from observational studies or case series, } \\
\text { or from RCTs with serious flaws }\end{array}$ \\
2A $\quad \begin{array}{l}\text { Consistent evidence from RCTs without important limitations, or exceptionally } \\
\text { strong evidence from observational studies }\end{array}$ \\
2B $\quad \begin{array}{l}\text { Evidence from RCTs with important limitations (inconsistent results, methodological } \\
\text { flaws), or very strong evidence from observational studies }\end{array}$ \\
2C $\quad \begin{array}{l}\text { Evidence for at least one critical outcome from observational studies or case series, } \\
\text { or from RCTs with serious flaws or indirect evidence }\end{array}$ \\
RCT = randomised controlled trial.
\end{tabular}




\section{Anticoagulation of pregnant patients with mechanical valves}

Anticoagulant therapy is essential because of the risk of valve thrombosis and systemic embolism (grade 1A). ${ }^{[3]}$

The ideal anticoagulant in pregnancy should be one that does not cross the placenta, to avoid teratogenicity and fetal haemorrhage, and one that can easily be reversed. Anticoagulants available for the management of pregnant patients with mechanical heart valves include:

- Vitamin K antagonists

- Unfractionated heparin (UFH)

- Low-molecular-weight heparin (LMWH).

These South African (SA) guidelines discuss the use of enoxaparin (Clexane; Sanofi Aventis), UFH and warfarin. (The recommendation to use enoxaparin only is based on the fact that adequate laboratory monitoring is available in SA for enoxaparin and not other LMWHs. The testing of LMWH is not interchangeable.) The choice of anticoagulant remains challenging, because both vitamin $\mathrm{K}$ antagonists and heparins may be associated with maternal and fetal adverse events. ${ }^{[3-5]}$ The choice of anticoagulation should be considered in conjunction with risk factors for thromboembolism (valve type, position and history of thromboembolism) and economic factors (availability and cost of the anticoagulants, access to laboratory testing and specialist care), as well as maternal preferences (Table 2). ${ }^{[1]}$

Pregnant patients with mechanical heart valves should be referred to a specialist antenatal clinic for management by a multidisciplinary team including an obstetrician and a cardiologist, with laboratory monitoring by a haematologist.

Table 2. Factors affecting choice of anticoagulant

Risk factors for thromboembolism
Valve type
Valve position
History of thromboembolism
Economic factors
Availability of the anticoagulants
Cost of the anticoagulants
Access to laboratory testing
Access to specialist care
Maternal preferences
Fetal and maternal adverse outcomes

\section{Anticoagulants 4.1 LMWH (enoxaparin)}

LMWH does not cross the placenta, and is associated with improved fetal outcomes.

In pregnant patients, $\mathrm{LMWH}$ at fixed doses is less effective than warfarin therapy in preventing thromboembolic complications. LMWH therefore requires dose adjustment with laboratory monitoring (grade 1A). ${ }^{[1]}$

LMWH offers definite advantages over UFH because of its longer plasma half-life, dose-dependent clearance (resulting in a more predictable anticoagulant response), improved side-effect profile and less frequent monitoring requirements (grade 1B). ${ }^{[6]}$

Allergic skin reactions can occur with LMWH, but are uncommon.

LMWH (enoxaparin) with monitoring and dose adjustment is strongly recommended throughout pregnancy (grade 1A).

\subsubsection{LMWH dosage}

The recommended dosage for enoxaparin is:

- Subcutaneous LMWH 12-hourly with dose adjustment to achieve a peak anti-Xa level $\sim 1 \mathrm{U} / \mathrm{mL}(1.0-1.2 \mathrm{U} / \mathrm{mL}) 3$ - 4 hours post injection. ${ }^{[1,2,7]}$

\subsubsection{Monitoring of therapy}

- LMWH activity is measured using an anti-Xa activity assay.
- Anti-Xa testing is performed by specialised National Health Laboratory Service central laboratories and private laboratories in SA (Table 3).

- A $5 \mathrm{~mL}$ citrate tube is required for the assay. Avoid sampling blood from indwelling lines.

- Samples must reach the reference laboratory (or local laboratory for shipping) within 1 hour.

- Samples must be drawn 3 - 4 hours after the last LMWH subcutaneous injection.

- Repeat testing is indicated until a therapeutic anti-Xa level is achieved (1.0 $1.2 \mathrm{U} / \mathrm{mL}){ }^{[2]}$

- A higher anti-Xa range than that proposed by international guidelines $(0.7-1.2 \mathrm{U} /$ $\mathrm{mL}$ ) is recommended owing to the high risk of valve thrombosis. ${ }^{[2,8]}$

- There is an increased dose requirement during pregnancy because of increased volume of distribution and renal clearance, and continued regular monitoring and dose adjustments (increase or decrease by $10 \mathrm{mg}$ ) according to anti-Xa levels are therefore recommended (Table 4). ${ }^{[7]}$

- Anti-Xa levels should be monitored weekly (as opposed to monthly) to reduce the likelihood of subtherapeutic anti-Xa levels and subsequent valve thrombosis. ${ }^{[8-10]}$

- The role of trough anti-Xa levels in adjusting the dose of $\mathrm{LMWH}$ is uncertain and pre-dose monitoring is not currently recommended.

\section{Table 3. Laboratories in SA that offer anti-Xa testing}

\begin{tabular}{ll}
\hline Laboratory & Contact number \\
\hline National Health Laboratory Service central laboratories & \\
Charlotte Maxeke Johannesburg Academic Hospital, Gauteng & $(011) 489-8534$ \\
Red Cross War Memorial Children's Hospital, Western Cape & $(021) 658-5203 / 4$ \\
Groote Schuur Hospital, Western Cape & $(021) 404-4151$ \\
Inkosi Albert Luthuli Central Hospital, KwaZulu-Natal (separated and \\
referred on ice to Ampath NRL) & $(031) 240-2677$ \\
Private laboratories & \\
Ampath & \\
$\quad \begin{array}{l}\text { NRL, Gauteng or } \\
\text { Separated and referred on ice to Ampath NRL }\end{array}$ & \\
Lancet & $(012) 678-0591$ \\
$\quad$ Milpark, Gauteng & \\
$\quad$ Pencardia, Gauteng & \\
Vermaak & \\
$\quad$ Unitas, Gauteng & $(011) 482-5406$ \\
Pathcare & (012) $483-0100$ \\
$\quad$ Cape Town, Western Cape &
\end{tabular}




\begin{tabular}{l} 
Table 4. Interpretation of anti-Xa levels in patients on LMWH \\
\hline Target anti-Xa levels \\
1.0 - 1.2 anti-Xa U/mL \\
Low anti-Xa level \\
Inadequate dosing \\
Delayed specimen draw \\
Dose of LMWH omitted \\
Weight gain \\
Gestation (volume of distribution of LMWH changes) \\
High anti-Xa level \\
Excessive dosing \\
Weight loss \\
Renal dysfunction \\
Reduced creatinine clearance (end of third trimester)
\end{tabular}

\subsubsection{Management of bleeding}

- LMWH (plasma half-life 3 - 6 hours) should be discontinued, as well as any other agents that may contribute to bleeding.

- Measurement of anti-Xa levels is suggested.

- One intravenous (IV) dose of protamine sulphate can neutralise about $60-75 \%$ of the anti-Xa activity. ${ }^{[4]}$

- $1 \mathrm{mg}$ protamine sulphate IV can be administered per $1 \mathrm{mg}$ (equivalent to 100 anti-Xa units) enoxaparin, given in the last dose over 10 minutes.

- If the patient continues to bleed, a repeat dose of $0.5 \mathrm{mg}$ protamine sulphate IV per $1 \mathrm{mg}$ of enoxaparin, up to a maximum of three doses, may be administered in conjunction with replacement therapy.

\subsection{UFH}

UFH does not cross the placenta and is associated with improved fetal outcome.

UFH at fixed doses is less effective than warfarin in preventing maternal thromboembolic complications. ${ }^{[9,11]}$ UFH therefore requires dose adjustment with frequent laboratory monitoring. Furthermore, the efficacy and dosage of adjusted-dose subcutaneous UFH has not been definitively established. The use of low-dose UFH is inadequate. ${ }^{[3]} \mathrm{LMWH}$ is recommended instead of UFH (grade 1B). ${ }^{[8]}$

Long-term UFH use is associated with an increased risk of heparin-induced thrombocytopenia (HIT), osteoporosis and allergic skin reactions.

UFH is not recommended in pregnant patients with mechanical heart valves (grade 1B).

\subsubsection{UFH dosage}

There is no consensus on the recommended dosage for UFH:

- Subcutaneous UFH in high doses 12-hourly with dose adjustment 6 hours post injection to achieve an activated partial thromboplastin time (aPTT) ratio of $2-2.5$ times the baseline value. ${ }^{[1,3,12]}$

\subsubsection{Monitoring of therapy}

- Anticoagulant activity is measured using an aPTT test.

- A $5 \mathrm{~mL}$ citrate tube is required for the assay. Avoid sampling blood from indwelling lines.

- Samples must reach the reference laboratory within 2 hours.
- Samples must be drawn 6 hours after the last UFH subcutaneous injection and repeated until a therapeutic aPTT is achieved (at least twice the baseline value).

- Once therapeutic levels are achieved, continued regular weekly monitoring with dose adjustments for changes with weight gain is recommended. UFH is more difficult to monitor owing to its unpredictable pharmacokinetics, and more frequent monitoring may be indicated.

- The patient's platelet count should be monitored on initiation of UFH and 7 days later while on therapy to assess for HIT. If the patient has been exposed to UFH in the past 100 days, a repeat platelet count is indicated within 24 hours of starting UFH.

\subsubsection{Management of bleeding}

- UFH (plasma half-life 1 - 2 hours) should be discontinued, as well as any other agents that may contribute to bleeding.

- Measurement of aPTT levels may be indicated.

- If bleeding is life-threatening, immediate reversal of UFH activity can be achieved by administration of protamine sulphate.

- $1 \mathrm{mg}$ of protamine sulphate IV should be administered per $1 \mathrm{mg}$ (equivalent to 100 units of heparin) given in the last subcutaneous dose over 10 minutes.

- Rapid infusion can cause hypotension and convulsions.

- Following IV administration, neutralisation occurs within 5 minutes. The maximum dose of protamine sulphate that can be administered is $50 \mathrm{mg}$.

\subsection{Warfarin}

Warfarin is the most effective anticoagulant for the prevention of valve thrombosis. ${ }^{[10]}$ Warfarin is easy to administer and monitor.

Warfarin is safe during the first 6 weeks of gestation, but is associated with a teratogenic effect between 6 and 12 weeks. This results in a $4-10 \%$ risk of warfarin embryopathy (Table 5). ${ }^{[2-3,13,14]} \mathrm{A}$ dose-dependent ( $<5 \mathrm{mg} / \mathrm{d}$ ) relationship for warfarin embryopathy has not been found in all cohorts. ${ }^{[15]}$ Warfarin is therefore not recommended in the first trimester (grade $1 \mathrm{~A}){ }^{[16-17]}$

In addition, there is an increased risk of fetal ocular and neurological abnormalities, a 30\% risk of fetal loss, and an increased risk of fetal intracranial haemorrhage at any time during pregnancy, but particularly at the time of delivery. ${ }^{[3,18-21]}$

In pregnant women at higher risk of thromboembolism, such as those with older-generation valve types in the mitral position, e.g. Starr-Edwards and Bjork-Shiley, or a history of thromboembolism on heparin, warfarin can be considered throughout pregnancy with replacement by UFH or LMWH at 36 weeks (grade 2C). ${ }^{[7]}$

Warfarin in the first trimester is not recommended (grade 1A).

\subsubsection{Warfarin dosage}

The recommended dosage for warfarin is:

- $5 \mathrm{mg}$ daily and the dose adjusted to achieve a target international normalised ratio (INR) of 3.0 (range $2.5-3.5$ ). ${ }^{[1]}$

\subsubsection{Monitoring of therapy}

- Anticoagulant activity is monitored using an INR test.

- A $5 \mathrm{~mL}$ citrate tube is required for the assay. Avoid sampling blood from indwelling lines.

- INR monitoring is indicated every 1 - 4 weeks. The dose should be adjusted to achieve a target INR of 3.0 (range 2.5 - 3.5).

- Fluctuations of the INR outside the desired target range should be investigated. 
4.3.3 Management of bleeding

- Women may go into preterm labour or develop complications requiring urgent delivery.

- Bleeding associated with warfarin therapy can be severe because of the drug's long plasma half-life of 40 hours.

- If bleeding is significant, warfarin activity can be reversed immediately by administration of fresh-frozen plasma (15 - $20 \mathrm{~mL} / \mathrm{kg} \mathrm{IV}$ ), prothrombin complex concentrates $(50 \mathrm{U} / \mathrm{kg})$ and/or vitamin $\mathrm{K}$ (1 - $2 \mathrm{mg}$ ) orally or intravenously. ${ }^{[22]}$

- Neonates born to mothers on warfarin will be over-anticoagulated, and caesarean section is the recommended mode of delivery.

- Assisted delivery with forceps and ventouse is contraindicated. ${ }^{[4]}$

- Maternal and neonatal INR monitoring is indicated daily.

\subsection{Aspirin}

The addition of low-dose aspirin in pregnant patients with mechanical heart valves is not routinely recommended (grade $2 \mathrm{C}$ ). ${ }^{[1]}$

\section{Management of pregnant patients with mechanical heart valves}

The suggested regimen for anticoagulation in pregnant patients with mechanical heart valves is set out in Fig. 1.

\subsection{Pre-pregnancy}

- A multidisciplinary team needs to be involved in the management of pregnancy,

\begin{tabular}{l} 
Table 5. Adverse effects of warfarin in \\
the fetus \\
\hline Warfarin embryopathy (6 - 12 weeks) \\
Nasal hypoplasia \\
Stippled epiphyses \\
Saddle-nose deformity \\
Mental retardation \\
Optic atrophy \\
Frontal bossing \\
Hypertelorism \\
High-arched palate \\
Short neck \\
Short stature \\
Fetal effects (all trimesters and delivery) \\
Ocular abnormalities - blindness \\
Neurological abnormalities - \\
microcephaly, mental retardation, low \\
intelligent quotients \\
Fetal loss \\
Bleeding
\end{tabular}

including an obstetrician, a cardiologist and a haematologist, with easy access to an anaesthetist, a paediatrician and a geneticist as the time of delivery approaches.

- Discuss the choice of anticoagulant with the patient, detailing the risks and benefits for all anticoagulant options and other cardiac medications (Table 2).

- Continue warfarin until pregnancy is achieved (grade 2C). ${ }^{[1]}$

- Advise patients to present early to a specialist antenatal clinic when menstruation is delayed.

\subsection{Antenatal}

The first visit to the antenatal clinic should include:

- A full history and examination, including cardiac, obstetric, haematological and anaesthetic assessment

- Baseline transthoracic echocardiography and 12-lead electrocardiography

- Antenatal bloods and INR (to exclude over-anticoagulation)

- A switch to subcutaneous LMWH, e.g. enoxaparin $1 \mathrm{mg} / \mathrm{kg}$ 12-hourly, is recommended in the first trimester once the
INR is $\leq 2.5$ and if there are no signs or symptoms of bleeding (grade $1 \mathrm{~A}) .^{[1]}$

- Adjust the dose of LMWH (by $10 \mathrm{mg}$ upwards or downwards) to achieve a peak anti-Xa level $\sim 1 \mathrm{U} / \mathrm{mL} 3$ - 4 hours post injection. Thereafter anti-Xa levels should be monitored, ideally on a weekly basis. $^{[8]}$

Follow-up antenatal visits (in addition to routine obstetric management) include:

- A history of bleeding, valve thrombosis (chest pain, shortness of breath), peripheral thromboembolic disease (focal neurological signs and symptoms)

- An examination of haematological (signs of bleeding, injection sites), cardiac (cardiac dysrythmias, congestive cardiac failure and auscultation to assess valve function), and neurological (signs of a cerebrovascular insult) systems

- Transthoracic echocardiography at the first antenatal visit and in the third trimester, or if clinically indicated to exclude valve thrombosis

- Continued adjusted-dose subcutaneous LMWH until 38 weeks is strongly recommended (grade 1A) (Table 6).

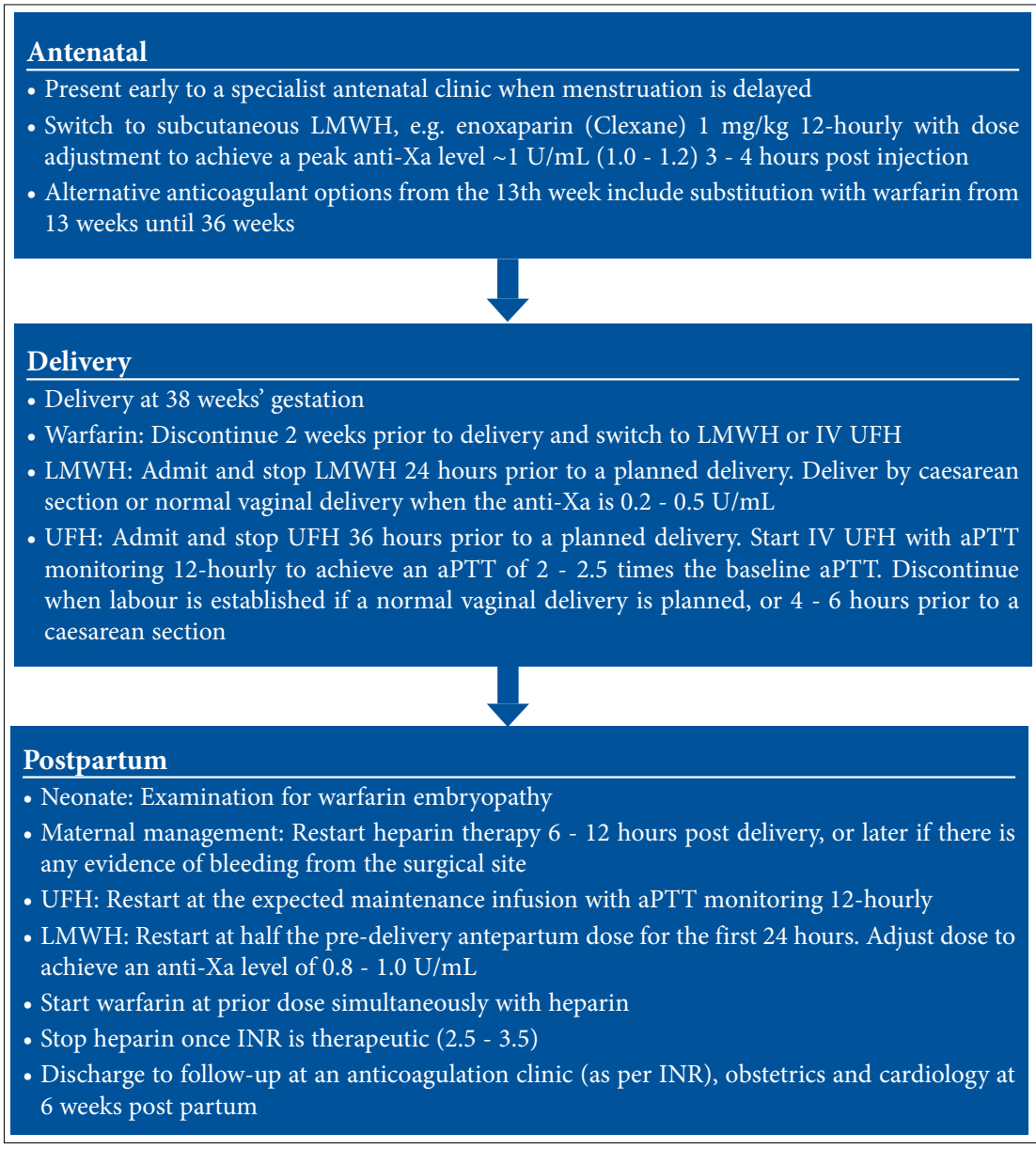

Fig. 1. Suggested regimen for anticoagulation in pregnant patients with mechanical heart valves. 
Table 6. Recommended anticoagulant regimens ${ }^{[1]}$

Adjusted-dose LMWH 12-hourly throughout pregnancy with dose adjustment to achieve a peak anti-Xa level $\sim 1 \mathrm{U} / \mathrm{mL} 3$ - 4 hours post injection (grade 1A) LMWH (as above) until the 13th week with substitution by warfarin until close to delivery, when UFH or LMWH is resumed (grade 1A)

In pregnant women at higher risk of thromboembolism, such as those with older-generation valve types in the mitral position or a history of thromboembolism on heparin, warfarin can be considered throughout pregnancy with replacement by UFH or LMWH close to delivery (grade 2C)

- Alternative anticoagulant options from the 13th week include substitution with warfarin from 13 weeks until 36 weeks (grade 1A) (Table 6).

- Close monitoring with dose adjustment is indicated. If the range is subtherapeutic and non-compliance is suspected, admission may be indicated.

\subsection{Delivery}

Delivery is planned at 38 weeks' gestation. The mode of delivery is determined by the obstetrician. ${ }^{[23]}$ In pregnant patients with HIV infection, specific guidelines should be consulted.

If warfarin is used, it should be discontinued 2 weeks prior to delivery and patients switched to LMWH or IV UFH. The fetal INR takes longer to normalise than the maternal INR, and delivery with the mother on warfarin poses a severe risk of haemorrhage to the fetus.

Suggested intrapartum anticoagulant protocols for delivery include:

\subsubsection{LMWH}

Suggested LMWH bridging regimen for peripartum anticoagulation:

- Admit and stop LMWH at least 24 hours prior to a planned delivery.

- Neuraxial anaesthesia should be avoided and epidural catheters should not be used (grade 1B).

- In order to reduce the risk of bleeding, anti-Xa levels should be used to guide the timing of delivery. ${ }^{[8]}$

- Perform serial anti-Xa testing 12 hours after the last dose of LMWH.

- Deliver by caesarean section or normal vaginal delivery when the anti-Xa level is $0.2-0.5 \mathrm{U} / \mathrm{mL}$.

- Post induction of labour, if the anti-Xa level is $<0.5 \mathrm{U} / \mathrm{mL}$ and the patient is not in the active phase of labour, delivery by caesarean section is recommended.

- Assess for major bleeding postpartum (resulting in a drop in the haemoglobin concentration by $\geq 2 \mathrm{~g} / \mathrm{dL}$, or bleeding requiring transfusion of at least two units of packed red blood cells).

\subsubsection{UFH}

Suggested UFH bridging regimen for peripartum anticoagulation: ${ }^{[4,24]}$

- Admit and stop anticoagulation at least 36 hours prior to a planned delivery.

- Start IV UFH 24 hours prior to delivery.

- Start IV UFH (5 000 - $10000 \mathrm{U}$ as an intravenous infusion in $200 \mathrm{~mL}$ normal saline at $33 \mathrm{~mL} / \mathrm{h}$ ).

- aPTT monitoring is indicated 12-hourly to monitor UFH. Aim to achieve an aPTT of $2-2.5$ times the baseline aPTT.

- Induction of labour: IV UFH therapy should be discontinued when labour is established. This should be individualised and determined by the risk of bleeding.

- Delivery by caesarean section: IV UFH therapy should be discontinued 4 - 6 hours prior to surgery.

- Assess for major bleeding postpartum (resulting in a drop in the haemoglobin concentration by $\geq 2 \mathrm{~g} / \mathrm{dL}$ or as bleeding requiring transfusion of at least two units of packed red blood cells).

\subsection{Post partum}

\subsubsection{Management of the neonate}

The neonate requires examination by a paediatrician and/or geneticist for warfarin embryopathy.

\subsubsection{Maternal management}

Early reinstitution of anticoagulant therapy in these patients, in order to reduce the time off anticoagulation, results in a high risk of primary and secondary postpartum haemorrhage. ${ }^{[19]}$

Heparin therapy with LMWH or UFH should be restarted 6 - 12 hours post delivery (see dosage below), or should be delayed if there is any evidence of bleeding from the surgical site.

Suggested postpartum regimens include:

\subsubsection{UFH}

- UFH should be restarted without a bolus, at no more than the expected maintenance infusion. ${ }^{[4]}$ The PTT should be checked 12 hours after restarting therapy to allow time for a stable anticoagulant response.

- IV UFH, with its short half-life, allows for more flexible anticoagulant control.

\subsubsection{LMWH}

- The anti-Xa level at delivery should guide the timing and dosing of LMWH. ${ }^{[8]}$

- After delivery, LMWH should be restarted at half the pre-delivery antepartum dose for the next 24 hours.

- The anti-Xa should be monitored 3 - 4 hours post therapeutic dose.

- Adjust the dose to achieve an anti-Xa level of $0.8-1.0 \mathrm{U} / \mathrm{mL}$.

Start warfarin at previous dose simultaneously with LMWH. Warfarin therapy should be delayed post delivery in order to reduce the risk of major bleeding. Stop LMWH once the INR is therapeutic (2.5 - 3.5).

Discharge, for follow-up at an anticoagulation clinic (as per INR), and for cardiology and obstetrics and gynaecology follow-up at 6 weeks post partum.

Declaration of conflicting interests. HRB reported having served as a scientific advisory board member for Sanofi-Aventis, Bayer HealthCare, Bristol-Myers Squibb, Daichi-Sankyo, GlaxoSmithKline, Pfizer, Roche, Isis and Thrombogenics, and received honoraria from Sanofi-Aventis, Bayer HealthCare, Bristol-Myers Squibb, DaichiSankyo, GlaxoSmithKline, Pfizer, Roche, Isis and Thrombogenics. SH reported having served as a scientific advisory board member for Bayer HealthCare, Bristol-Myers Squibb, Daiichi-Sankyo and Sanofi-Aventis, and received honoraria from Aspen, Bayer Healthcare, Bristol-Myers Squibb, Daiichi-Sankyo and Pfizer. BFJ has received honoraria from Bayer HealthCare, Boehringer and Sanofi-Aventis. RSC reported having served as a scientific advisory board member for Sanofi-Aventis, and has received honoraria from Pfizer, Sanofi-Aventis, Bayer HealthCare and LEO Pharma. 
1. Bates SM, Greer IA, Middeldorp S, et al. VTE, thrombophilia, antithrombotic therapy, and pregnancy: Antithrombotic Therapy and Prevention of Thrombosis, 9th ed: American College of Chest Physicians evidence-based clinical practice guidelines. Chest 2012;141(2 Suppl):e691S-e736S. [http://dx.doi. org/10.1378/chest.11-2300]

2. Bonow RO, Carabello BA, Chatteriee K, et al. ACC/AHA 2006 guidelines for the management of patients with valvular heart disease: A report of the American College of Cardiology/American Heart Association Task Force on Practice Guidelines (writing Committee to Revise the 1998 guidelines fo the management of patients with valvular heart disease) developed in collaboration with the Society of Cardiovascular Anesthesiologists endorsed by the Society for Cardiovascular Angiography an Interventions and the Society of Thoracic Surgeons. J Am Coll Cardiol 2006;48(3):e1-e148. [http:// dx.doi.org/10.1016/j.jacc.2006.05.021]

3. Chan WS, Anand S, Ginsberg JS. Anticoagulation of pregnant women with mechanical heart valves: A systematic review of the literature. Arch Intern Med 2000;160(2):191-196. [http://dx.doi.org/10.1001/ archinte.160.2.191]

4. McLintock C. Anticoagulant therapy in pregnant women with mechanical prosthetic heart valves No easy option. Thromb Res 2011;127(Suppl 3):S56-S60. [http://dx.doi.org/10.1016/S00493848(11)70016-0]

5. Ginsberg JS, Chan WS, Bates SM, Kaatz S. Anticoagulation of pregnant women with mechanical heart valves. Arch Intern Med 2003;163(6):694-698. [http://dx.doi.org/10.1001/archinte.163.6.694]

6. Greer IA, Nelson-Piercy C. Low-molecular-weight heparins for thromboprophylaxis and treatment 6. Greer IA, Nelson-Piercy C. Low-molecular-weight heparins for thromboprophylaxis and treatment
of venous thromboembolism in pregnancy: A systematic review of safety and efficacy. Blood of venous thromboembolism in pregnancy: A systematic review
2005;106(2):401-407. [http://dx.doi.org/10.1182/blood-2005-02-0626]

7. Saeed CR, Jacobson BF, Pravin M, Aziz RH, Serasheini M, Dominique TG. A prospective trial showing the safety of adjusted-dose enoxaparin for thromboprophylaxis of pregnant women with mechanical prosthetic heart valves. Clin Appl Thromb Hemost 2011;17(4):313-319. [http://dx.doi. org/10.1177/1076029610371470]

8. Salazar E, Izaguirre R, Verdejo J, Mutchinick O. Failure of adjusted doses of subcutaneous heparin to prevent thromboembolic phenomena in pregnant patients with mechanical cardiac valve prostheses. I Am Coll Cardiol 1996;27(7):1698-1703. [http://dx.doi.org/10.1016/0735-1097(96)00072-1]

9. Al-Lawati AA, Venkitraman M, Al-Delaime T, Valliathu J. Pregnancy and mechanical heart valves replacement: Dilemma of anticoagulation. Eur J Cardiothorac Surg 2002;22(2):223-227. [http://dx.doi. org/10.1016/S1010-7940(02)00302-0]

10. Regitz-Zagrosek V, Blomstrom Lundqvist C, et al. ESC Guidelines on the management of cardiovascular diseases during pregnancy: The Task Force on the Management of Cardiovascular Diseases during Pregnancy of the European Society of Cardiology (ESC). Eur Heart J 2011;32(24):3147-3197. [http:// dx.doi.org/10.1093/eurhearti/ehr218]

11. Geelani MA, Singh S, Verma A, Nagesh A, Betigeri V, Nigam M. Anticoagulation in patients with mechanical valves during pregnancy. Asian Cardiovasc Thorac Ann 2005;13(1):30-33. [http://dx.do org/10.1177/021849230501300107]
12. Guidozzi F. Pregnancy in patients with prosthetic cardiac valves. S Afr Med J 1984;65(24):961-

13. Hall JG, Pauli RM, Wilson KM. Maternal and fetal sequelae of anticoagulation during pregnancy. Am J Med 1980;68(1):122-140. [http://dx.doi.org/10.1016/0002-9343(80)90181-3]

14. Sareli P, England MJ, Berk MR, et al. Maternal and fetal sequelae of anticoagulation during pregnancy in patients with mechanical heart valve prostheses. Am J Cardiol 1989;63(20):1462-1465. [http:// dx.doi.org/10.1016/0002-9149(89)90008-8

15. Vitale N, de Feo M, de Santo LS, Pollice A, Tedesco N, Cotrufo M. Dose-dependent fetal complication of warfarin in pregnant women with mechanical heart valves. J Am Coll Cardiol 1999;33(6):1637-1641 [http://dx.doi.org/10.1016/S0735-1097(99)00044-3]

16. Mazibuko B, Ramnarain H, Moodley J. An audit of pregnant women with prosthetic heart valves at a tertiary hospital in South Africa: A five-year experience. Cardiovasc J Afr 2012;23(4):216-221. [http:// dx.doi.org/10.5830/CVJA-2012-022]

17. Soma-Pillay P, Mathivha T, Macdonald P. Correlation between warfarin dose and maternal and fetal outcomes in pregnant women with prosthetic heart valves. Presented at the 1st International Conference on Cardiac Problems in Pregnancy, Valencia, Spain, 20-25 February 2010.

18. Elkayam U, Singh H, Irani A, Akhter MW. Anticoagulation in pregnant women with prosthetic heart valves. J Cardiovasc Pharmacol Ther 2004;9(2):107-115. [http://dx.doi.org/10. etic heart valves. $1177 / 107424840400900206]$

19. McLintock C, McCowan LM, North RA. Maternal complications and pregnancy outcome in women with mechanical prosthetic heart valves treated with enoxaparin. BJOG 2009;116(12):1585-1592 [http://dx.doi.org/10.1111/j.1471-0528.2009.02299.x]

20. Shannon MS, Edwards MB, Long F, Taylor KM, Bagger JP, de Swiet M. Anticoagulant management of pregnancy following heart valve replacement in the United Kingdom, 1986-2002. J Heart Valve Dis 2008;17(5):526-532.

21. Topol EJ. Anticoagulation with prosthetic cardiac valves. Arch Intern Med 2003;163(18):2251-2252 [http://dx.doi.org/10.1001/archinte.163.18.2251]

22. Schulman S, Beyth RJ, Kearon C, Levine MN. Hemorrhagic complications of anticoagulan and thrombolytic treatment: American College of Chest Physicians Evidence-Based Clinical Practice Guidelines (8th Edition). Chest 2008;133(6 Suppl):257S-298S. [http://dx.doi.org/10.1378/ chest.08-0674]

23. Pieper PG. The pregnant woman with heart disease: Management of pregnancy and delivery. Neth Heart J 2012;20(1):33-37. [http://dx.doi.org/10.1007/s12471-011-0209-y]

24. Bates SM. Management of pregnant women with thrombophilia or a history of venous thromboembolism. ASH Education Book 2007;1:143-150. [http://dx.doi.org/10.1182/asheducation2007.1.143]

Accepted 20 July 2015. 